\title{
Molecular characterization of mutation in the parC and gyrB genes that confer fluoroquinolone resistance in Streptococcus pneumonia isolates
}

\author{
Laila M. Yousif ${ }^{1}$, Ghada A. Ismael ${ }^{2}$, Ashraf k. Mohammed ${ }^{3}$ and Mohammed H. \\ Mahmoud $^{4}$ \\ Department of Clinical Pathology, Faculty of Medicine, Sohag University ${ }^{1,3,4}$ \& Ain \\ Shams University ${ }^{2}$
}

\section{Abstract}

Resistance of Streptococcus pneumoniae to multiple antibacterial agents, including $\beta$-lactams, macrolides, tetracyclines, and co-trimoxazole, has emerged worldwide in the 1980s and 1990s and has emphasized the need for new therapeutic alternatives, such as newer fluoroquinolones. Older fluoroquinolones, such as ciprofloxacin and ofloxacin, have been widely used in the last 2 decades, but their activity against gram-positive pathogens is limited. Newer fluoroquinolones, such as levofloxacin, gatifloxacin, moxifloxacin, and gemifloxacin, have enhanced activity against most respiratory pathogens, and some are being more widely used to treat respiratory tract infections. Therefore, the emergence of fluoroquinoloneresistant S. pneumoniae strains, although worldwide prevalence is low, is a concern to clinicians who manage respiratory tract infections.

Aim of the work: The aim of this study was to determine the prevalence of fluoroquinolone resistance Streptococcus pneumoniae (FQRSP) and to examine the genetic relatedness of pneumococcal isolates with parC and gyrB genes mutations in different specimens.

Patients and Methods: In this study, Biometra Thermal Cyclar-T Gradient Software PCR system version 4 together with DNASIS 2.6 Sequence Analysis Programs were used to investigate the presence of mutations at quinolone resistance-determining regions of topoisomerase IV and DNA gyrase on $78 \mathrm{~S}$. pneumoniae strains, Among 78 isolates 37 (47.4\%) of S. pneumonia isolates were Fluroquinolones susceptible, $12(15.4 \%)$ were with variable susceptibility and 29 (37.2\%) were Fluroquinolones resistant.

Results: Our study illustrate the role of mutation in the parC \& gyrB genes and the effect of mutations in the both genes in fluoroquinolone resistance among $\mathrm{S}$. pneumoniae isolates.

Conclusion: Results indicated that there is a significant correlation between quinolone resistance development and mutations in the $\operatorname{parC}$ gene and in less significance in the $\operatorname{gyr} B$ genes .

Key words: GyrB, ParC, Streptococcus pneumonia, Fluoroquinolones.

\section{Introduction}

Antimicrobial resistance (AMR) is the ability of a microbe to resist the effects of medication previously used to treat them. This broader term also covers antibiotic resistance, which applies to bacteria and antibiotics. Resistance arises through one of three ways: natural resistance in certain types of bacteria, genetic mutation, or by one species acquiring resistance from another. Resistance can appear spontaneously because of random mutations; or more commonly following gradual buildup over time, and because of misuse of antibiotics or antimicrobials. Resistant microbes are increasingly difficult to treat, requiring alternative medications or higher doses, both of which may be more expensive or more toxic. Microbes resistant to multiple antimicrobials are called multidrug resistant (MDR); or sometimes superbugs. Antimicrobial resistance is on the rise with millions of deaths every year. All classes of microbes 
develop resistance: fungi develop antifungal resistance, viruses develop antiviral resistance, protozoa develop antiprotozoal resistance, and bacteria develop antibiotic resistance ${ }^{(3)}$.

The rise in gram-positive pathogen resistance in recent years has prompted the pharmaceutical industry to develop fluoroquinolones with greater activity against these rapidly changing pathogens. Structural modifications to the basic fluoroquinolone nucleus have given rise to several new generations of compounds. With each new generation the potency against many gram-positive pathogens, including S. pneumoniae, has improved. Although the worldwide prevalence of fluoroquinolone-resistant S. pneumoniae remains low in relation to $\beta$-lactam resistance $(<1 \%)$, the dissemination of successful resistant clones has nonetheless increased the prevalence in some countries ${ }^{(4)}$.

Resistance to fluoroquinolones in $\mathrm{S}$. pneumoniae arises in a stepwise fashion and results from alterations in the target binding site due to the acquisition of spontaneous mutations in the quinolone resistancedetermining regions (QRDRs) of the topoisomerase IV and DNA gyrase genes. Although mutations usually occur in the QRDRs of parE and gyrA, a role for mutations in the parE and gyrA subunits in low-level resistance has been reported ${ }^{(5)}$.

$\mathrm{S}$. pneumoniae isolates with a mutation only in ParC usually remain susceptible or display only a modest increase in resistance. But these firststep mutants are associated with an increased risk for secondary mutations that may enhance resistance. Higher levels of fluoroquinolone resistance require mutations in parC. Resistance to fluoroquinolones can also occur through the overexpression of efflux systems, a phenotype frequently observed among resistant clinical pneumococci. During the past few years, Fluoroquinolone-Resistant Streptococcus pneumoniae (FQRSP) has been reported from several countries, although the prevalence remains low. Fluoroquinolone nonsusceptibility among pneumococci results mainly from point mutations in the quinolone resistance-determining regions QRDR topoisomerase genes (6).

Inappropriate use of any antibiotic can contribute to the emergence of resistance to that and related agents. So much work is needed to identify optimal strategies to prevent the emergence and spread of resistant pneumococcal strains in long-term care facilities, including potential use of pneumococcal conjugate vaccines, antimicrobial stewardship, and infection control interventions to interrupt transmission (6)

\section{Aim of the work}

The aim of this study was to determine the prevalence of fluoroquinolone resistance Streptococcus pneumoniae (FQRSP) and to examine the genetic relatedness of pneumococcal isolates with parC and gyrB genes mutations in different specimens.

\section{Patients and Methods}

This study was prospectively conducted over a period of 24 months between October 2015 and September 2017, at Sohag university hospital. During the study period, 78 patients hospitalized for a syndrome consistent with a diagnosis of CAP (community acquired pneumonia) included in this study with a mean age of 34.5 years (range, 2 to 67 ), $60 \%$ of whom were males. 


\section{Clinical specimens.}

Fresh sputum samples were collected soon after collection of data from patients (75 specimens). Representative sputum originating from the lower respiratory tract was defined as that containing $>25$ granulocytes and $<10$ epithelial cells per low power field (lpf: total magnification: $\times 100$ ).

Bronchoalveolar lavage (BAL) (3 specimens) as diagnostic techniques were used according to the clinical judgment of the physician in charge for some neonates.

\section{Identification of Pneumococci and} antimicrobial susceptibility testing.

Isolates were incubated in plates with increased $\mathrm{CO} 2(5-10 \%)$ in order to enhance the development of hemolytic zones of the pathogenic Streptococci and incubated for 18-24 hours. On a blood agar plate (BAP), colonies of S. pneumoniae appear as small, grey, moist (sometimes mucoidal), glistening round colonies, about $1 \mathrm{~mm}$ in diameter, and surrounded by a zone of alphahemolysis.

By gram stain isolates appear as lancet-shaped, Gram-positive diplococci or chains of cocci. The identification of bacteria in our samples was completed by the VITEK ${ }^{\circledR} 2$ Compact System. As a commercial and standard system, its accuracy has been strictly evaluated.

Antimicrobial susceptibility testing and the MIC was determined by using VITEK ${ }^{\circledR} \quad 2 \quad$ Antimicrobial Susceptibility Tests (AST) according to the Clinical and Laboratory Standards Institute (CLSI).

\section{Polymerase chain reaction $(\mathrm{PCR})$ :-}

Simple PCR was performed for all strains to detect Fluoroquinolone Resistance Streptococcus pneumoniae (FQRSP) and to examine the genetic relatedness of pneumococcal isolates with par C, gyrB, parE and gyrA genes mutations.

\section{i) Bacterial DNA extraction.}

By the use of Quick-DNA ${ }^{\text {TM }}$ Miniprep Kit (Catalog No D3024). Bacterial genomic DNA was extracted from several colonies of each isolate and extracted DNA was stored at $20 \circ \mathrm{C}$ prior to PCR amplification.

ii) DNA amplification.

The QRDRs of gyrA, gyrB, parC, and parE were amplified by Biometra Thermal Cyclar-T Gradient Software PCR system version 4. The primers for each loci were synthetized by (metabion international $\mathrm{AG}$, Germany) as described by Maeda et al. 2011. The primers used For amplification gyrA gene, nucleotide sequence as follow:

F2-

GACAAAGGAGATGAAGGCAAG, R2-GAAAATCTGGTCCAGGCAAG and for amplification gyrB gene: FGGGAAATAGCGAAGTGGTCA, RGTACGAATGTGGGCTCCAT and for amplification parC gene, FCAAAACATGTCCCTGGAGGA, RGCAGCATCTATGACCTCAGC and for amplification parE gene,:FTCAAGTCTGCCATTACCAAG G,RACCCGCACCAATGGTATAAA. PCR on lysates with primers as above using MyTaq ${ }^{\text {TM }}$ Red Mix, 2x (Bioline USA Inc., USA) was performed as follows: 1 cycle $95^{\circ} \mathrm{C}$ for $15 \mathrm{~min}$ for initial denaturation , 40 cycles of $94^{\circ} \mathrm{C}$ for $1 \mathrm{~min}, 56^{\circ} \mathrm{C}$ for $1 \mathrm{~min}, 72^{\circ} \mathrm{C}$ for 1 min, followed by an extension step of $72^{\circ} \mathrm{C}$ for $10 \mathrm{~min}$. The PCR products were separated by electrophoresis in a $2 \%$ agarose gel.

\section{iii) DNA sequencing}

Amplification products were purified with the QIAquick PCR purification kit (Qiagen). To identify mutations 
DNA Sequencing reactions were prepared with ABI Prism ${ }^{\circledR}$ BigDye Terminator Cycle Sequencing Ready Reaction Kit using conditions descriped by Zhanel et al. (8) with ABI 377 automated sequencer (PE Applied Biosystems, mississauga, ON).

\section{Sequence analysis}

DNA sequences were analyzed with DNASIS 2.6 Sequence Analysis Programs (Hitachi Software Engineering Co., Ltd., San Francisco, Calif.) against 1 of the 2 identical sequenced pneumococcal strains in the database (NC_008533 Streptococcus pneumoniae D39 and AE007317). D39 is a historically important serotype 2 strain that was used in experiments by Avery and coworkers to demonstrate that DNA is the genetic material.
Although isolated nearly a century ago, D39 remains extremely virulent in murine infection models and is perhaps the strain used most frequently in current studies of pneumococcal pathogenesis. To date, the complete genome sequences have been reported for only two S. pneumoniae strains: TIGR4 (The Institute for Genomic Research), a recent serotype 4 clinical isolate that derived from blood of a male Norwegian patient in 2001. Tettelin et al sequenced the complete genome of strain TIGR4 (9), and laboratory strain R6, an avirulent, unencapsulated derivative of strain D39 that was used in laboratory in 2001 by Hoskins et al (10).

\section{Results}

During the period from October 2015 and September 2017 our study was carried out in the Clinical Pathology Department, faculty of medicine, Sohag University Hospital, 78 participants included in our study, S. pneumoniae was isolated from 78 patients included in this study. The resistance percentages of all strains to tested antibiotics were as follows: $91 \%$ of isolates in our study were resistant to Ampicillin, 5.1\% were intermediate and $3.8 \%$ were susceptible. Regarding Cefaclor $83.3 \%$ were resistant, $7.7 \%$ were intermediate and $9 \%$ were susceptible. Erythromycin was resistant in $82.1 \%$ of isolates, intermediate in $10.3 \%$, and susceptible in $7.7 \%$. Regarding Imipenem $10.3 \%$ of isolates were resistant. Tetracycline was resistant in $71.8 \%$ of isolates. Clarithromycin was resistant in only $6.4 \%$, also $10.3 \%$ of our isolates were resistant to ceftriaxone. Trimethoprim/ sulfamethoxazole was resistant in $9 \%$ of our isolates.(table 1) 
SOHAG MEDICAL JOURNAL

Vol. 22 No.1 Jan 2018
Molecular characterization of mutation in the parC Mohammed H. Mahmoud 4

Table (1) Various antibiotics susceptibility of S. pneumoniae.

\begin{tabular}{|c|c|c|}
\hline Variable & MIC (ug/dl) & No $(\%)$ \\
\hline \multirow{3}{*}{ Ampicillin } & Resistant $\geq 2$ & $71(91 \%)$ \\
\hline & Intermediate $0.12-1$ & $4(5.1 \%)$ \\
\hline & Susceptible $\leq 0.06$ & $3(3.8 \%)$ \\
\hline \multirow{3}{*}{ Cefaclor } & Resistant $>16$ & $65(83.3 \%)$ \\
\hline & Intermediate $8-16$ & $6(7.7 \%)$ \\
\hline & Susceptible $\leq 4$ & $7(9 \%)$ \\
\hline \multirow{3}{*}{ Erythromycin } & Resistant $\geq 1$ & $64(82.1 \%)$ \\
\hline & Intermediate 0.5 & $8(10.3 \%)$ \\
\hline & Susceptible $\leq 0.25$ & $6(7.7 \%)$ \\
\hline \multirow{3}{*}{ Imipenem } & Resistant $\geq 1$ & $8(10.3 \%)$ \\
\hline & Intermediate 0.5 & $4(5.1 \%)$ \\
\hline & Susceptible $\leq 0.25$ & $66(84.6 \%)$ \\
\hline \multirow{3}{*}{ Tetracycline } & Resistant $\geq 8$ & $56(71.8 \%)$ \\
\hline & Intermediate 4 & $3(3.8 \%)$ \\
\hline & Susceptible $\leq 2$ & $19(24.4 \%)$ \\
\hline \multirow{3}{*}{ Clarithromycin } & Resistant $\geq 2$ & $5(6.4 \%)$ \\
\hline & Intermediate 1 & $3(3.8 \%)$ \\
\hline & Susceptible $\leq 0.5$ & $70(89.8 \%)$ \\
\hline \multirow{3}{*}{ Ceftriaxone } & Resistant $\geq 2$ & $8(10.3 \%)$ \\
\hline & Intermediate 1 & $4(5.1 \%)$ \\
\hline & Susceptible $\leq 0.5$ & $66(84.6 \%)$ \\
\hline \multirow{3}{*}{ Trimethoprim/ sulfamethoxazole } & Resistant $\geq 4$ & $7(9 \%)$ \\
\hline & Intermediate $1-2$ & $3(3.8 \%)$ \\
\hline & Susceptible $\leq 0.5$ & $68(87.2 \%)$ \\
\hline
\end{tabular}


SOHAG MEDICAL JOURNAL Vol. 22 No.1 Jan 2018
Molecular characterization of mutation in the parC

Mohammed H. Mahmoud ${ }^{4}$

Break points of antibiotics "Ampicillin" $\geq 2 \mathrm{R} \& 0.12-1 \quad \mathrm{I} \& \leq 0.06 \mathrm{~S}$, "Cefaclor" > $16 \mathrm{R} \& 8-16$ I \& $\leq 4 \mathrm{~S}$, "Erythromycin" $\geq 1 \mathrm{R} \& 0.5$ I \& $\leq 0.25 \mathrm{~S}$, "Imipenem" $\geq 1 \mathrm{R} \& 0.5 \mathrm{I} \& \leq 0.25 \mathrm{~S}$ "Tetracyclin" $\geq 8 \mathrm{R} \& 4 \mathrm{I} \& \leq 2 \mathrm{~S}$, "Clarithromycin" $\geq 2 \mathrm{R} \& 1$ I \& $\leq 0.5 \mathrm{~S}$, "Ceftriaxone" $\geq 2 \mathrm{R} \& 1 \mathrm{I} \& \leq 0.5 \mathrm{~S}$ and "Trimethoprim-Sulfamethaxzole " $\geq 4 \mathrm{R} \& 1-2$ I \& $\leq 0.5 \mathrm{~S}$.

Among 78 isolates $37(47.4 \%)$ of S. pneumonia isolates were Fluroquinolones susceptible $12(15.4 \%)$ were with variable susceptibility and $29(37.2 \%)$ were Fluroquinolones resistant.

The MICs of Ciprofloxacin, Levofloxacin, Gatifloxacin and Moxifloxacin were measured and results were as follow, $44.9 \%$ of S. pneumonia isolates were resistant to ciprofloxacin, $11.5 \%$ were intermediate and $43.6 \%$ were sensitive. Regarding levofloxacin $42.3 \%$ of isolates were resistant, $9 \%$ were intermediate, and $48.7 \%$ were sensitive. Over forty six (46.1\%) of our isolates were resistant to Gatfloxacin, $10.3 \%$ were intermediate, and $43.6 \%$ were sensitive. Regarding Moxifloxacin $46.2 \%$ of our isolates were resistant, $7.6 \%$ were intermediate, and $46.2 \%$ were sensitive (Table 2). Break points of fluroquinolones group "Ciprofloxacin" $\geq 4 \mathrm{R} \& 2 \mathrm{I} \& \leq 1 \mathrm{~S}$ and "Levofloxacin" $\geq 8 \mathrm{R} \& 4$ I \& $\leq 2 \mathrm{~S}$ and "Gatfloxacin" $\geq 4 \mathrm{R} \& 2 \mathrm{I} \& \leq 1 \mathrm{~S}$ "Moxifloxacin" $\geq 4 \mathrm{R} \& 2$ I \& $\leq 1 \mathrm{~S}$.

Table (2) Fluroquinolones susceptibility of S. pneumoniae.

\begin{tabular}{|c|c|c|}
\hline Variable & & no $(\%)$ \\
\hline \multirow{3}{*}{$\begin{array}{c}\text { Ciprofloxacin } \\
\left(1^{\text {st }} \text { generation Fluoroquinolone }\right)\end{array}$} & Resistant $\geq 4$ & $35(44.9 \%)$ \\
\hline & Intermediate 2 & $9(11.5 \%)$ \\
\hline & Susceptible $\leq 1$ & $34(43.6 \%)$ \\
\hline \multirow{3}{*}{$\begin{array}{c}\text { Levofloxacin } \\
\left(2^{\text {nd }} \text { generation Fluoroquinolone }\right)\end{array}$} & Resistant $\geq 8$ & $33(42.3 \%)$ \\
\hline & Intermediate 4 & $7(9 \%)$ \\
\hline & Susceptible $\leq 2$ & $38(48.7 \%)$ \\
\hline \multirow{3}{*}{$\begin{array}{c}\text { Gatfloxacin } \\
\left(3^{\text {rd }} \text { generation Fluoroquinolone }\right)\end{array}$} & Resistant $\geq 4$ & $36(46.1 \%)$ \\
\hline & Intermediate 2 & $8(10.3 \%)$ \\
\hline & Susceptible $\leq 1$ & $34(43.6 \%)$ \\
\hline \multirow{3}{*}{$\begin{array}{c}\text { Moxifloxacin } \\
\left(4^{\text {th }} \text { generation Fluoroquinolone }\right)\end{array}$} & Resistant $\geq 4$ & $36(46.2 \%)$ \\
\hline & Intermediate 2 & $6(7.6 \%)$ \\
\hline & Susceptible $\leq 1$ & $36(46.2 \%)$ \\
\hline
\end{tabular}

Sequencing of the Quinolone Resistance-Determining (QRDRs). $\quad$.I

Of the 41 quinolone resistant and intermediate isolates, 9 (22.0\%) had no substitutions in the QRDRs of either GyrB or ParC, 21 (51.2\%) had a QRDR ParC substitution, while $16(39.0 \%)$ had QRDRs substitutions in GyrB. The specific substitutions observed in ParC included: Ser79Ala, Ser79Phe, Ser79Tyr, and Asp83Asn. The combinations of substitutions observed in GyrA and ParC included: Ser81Phe and Ser79Phe, Ser81Tyr and Ser79phe, Glu85Lys and Ser79Phe, Ser81Phe and Ser79Tyr, Ser81Phe and Asp83Asn, the specific substitutions observed in GyrB were Ala583Ser and Arg545Asn The percent of isolates with each of the aforementioned substitutions is presented in table (3). 
SOHAG MEDICAL JOURNAL

Vol. 22 No.1 Jan 2018
Molecular characterization of mutation in the parC

Mohammed H. Mahmoud ${ }^{4}$

Table (3) Percent and types of the 41 Fluroquinolones-resistant $\mathrm{S}$. pneumoniae isolates with resistance-associated QRDR substitutions.

\begin{tabular}{|c|c|c|}
\hline \multicolumn{2}{|c|}{ Variable } & no (\%) \\
\hline \multirow{3}{*}{ GyrB } & Ala583Ser & $5(12.2 \%)$ \\
\cline { 2 - 3 } & \multirow{2}{*}{ Arg545Asn } & $3(7.3 \%)$ \\
\hline \multirow{4}{*}{ ParC } & Asp83Asn & $2(4.9 \%)$ \\
\cline { 2 - 3 } & Ser79Ala & $1(2.4 \%)$ \\
\cline { 2 - 3 } & Ser79Phe & $14(34.1 \%)$ \\
\cline { 2 - 3 } & Ser79Tyr & $4(9.8 \%)$ \\
\cline { 2 - 3 } & &
\end{tabular}

The most common ParC substitutions occurred at position Ser79, with Ser79Phe observed most frequently (34.1\% of all resistant isolates).

As shown in table 4, at Ciprofloxacin MIC 2, 4, 8 and 16, 75\%, 66.7\%, 77.8\% and $80 \%$ had no substitution in Gyr B, 22.2\%, $11.1 \%$ and 20\% at MIC 4, 8 and 16 had Ala583Ser substitution. 25\%, $11.1 \%$ and $11.1 \%$ at MIC 2, 4 and 8 had Arg545Asn substitution.

Regarding Par C, at Ciprofloxacin MIC 2, 4, 8 and 16, 75\%, 55.6\%, 33.3\% had no substitution in Par C, $11.1 \%$ and $10 \%$ at MIC 8 and 16 had Asp83Asn substitution. Only $10 \%$ at MIC 16 had Ser79Ala substitution. $25 \%, 33.3 \%, 33.3 \%$ and $70 \%$ at MIC 2, 4, 8 and 16 had Ser79Phe substitution. At MIC 4, 8 and 16, 11.1, 22.2\% and 10\% had Ser79Tyr substitution.

Table (4) MICs and substitutions observed in GyrB and ParC in Ciprofloxacin resistant and intermediate $S$. pneumoniae isolates.

\begin{tabular}{|c|c|c|c|c|}
\hline Variable (MIC) & $\mathbf{2}$ & $\mathbf{4}$ & $\mathbf{8}$ & $\mathbf{1 6}$ \\
\hline GyrB & & & & \\
\hline No substitution & $3(75 \%)$ & $6(66.7 \%)$ & $7(77.8 \%)$ & $8(80 \%)$ \\
\hline Ala583Ser & $0(0 \%)$ & $2(22.2 \%)$ & $1(11.1 \%)$ & $2(20 \%)$ \\
\hline Arg545Asn & $1(25 \%)$ & $1(11.1 \%)$ & $1(11.1 \%)$ & $0(0 \%)$ \\
\hline Par C & & & & $0(0 \%)$ \\
\hline No substitution & $3(75 \%)$ & $5(55.6 \%)$ & $3(33.3 \%)$ & $1(10 \%)$ \\
\hline Asp83Asn & $0(0 \%)$ & $0(0 \%)$ & $1(11.1 \%)$ & $1(10 \%)$ \\
\hline Ser79Ala & $0(0 \%)$ & $0(0 \%)$ & $0(0 \%)$ & $7(70 \%)$ \\
\hline Ser79Phe & $1(25 \%)$ & $3(33.3 \%)$ & $3(33.3 \%)$ & $1(10 \%)$ \\
\hline Ser79Tyr & $0(0 \%)$ & $1(11.1 \%)$ & $2(22.2 \%)$ & \\
\hline
\end{tabular}

As shown in table (5) at Levofloxacin MIC 4, 8 and 16, 75\%, 61.5\% and $85.7 \%$ had no substitution in Gyr B, 25\%, 23.1\% and 7.1\% at MIC 4, 8 and 16 had Ala583Ser substitution. $15.4 \%$ and $7.1 \%$ at MIC 4 and 8 had Arg545Asn substitution.

Regarding Par C, at Levofloxacin MIC 4, 8 and 16, 75\%, 30.7\%, 14.3\% had no substitution in Par C, 7.7\% and 7.1\% at MIC 8 and 16 had Asp83Asn substitution. Only $7.7 \%$ at MIC 8 had Ser79Ala substitution. 25\%, 38.5\% and 57.1\% at MIC 4, 8 and 16 had Ser79Phe substitution. At MIC 8 and 16, 15.4 and 14.4\% had Ser79Tyr substitution. Only $7.1 \%$ at MIC 16 had Ser81Phe substitution. 
SOHAG MEDICAL JOURNAL

Vol. 22 No.1 Jan 2018
Molecular characterization of mutation in the parC

Mohammed H. Mahmoud ${ }^{4}$

Table (5) MICs and substitutions observed in GyrB and ParC in Levofloxacin-resistant and intermediate S. pneumoniae isolates.

\begin{tabular}{|c|c|c|c|}
\hline Variable (MIC) & $\mathbf{4}$ & $\mathbf{8}$ & $\mathbf{1 6}$ \\
\hline GyrB & & & $12(85.7 \%)$ \\
\hline No substitution & $3(75 \%)$ & $8(61.5 \%)$ & $1(7.1 \%)$ \\
\hline Ala583Ser & $1(25 \%)$ & $3(23.1 \%)$ & $1(7.1 \%)$ \\
\hline Arg545Asn & $0(0 \%)$ & $2(15.4 \%)$ & $2(14.3 \%)$ \\
\hline Par C & & & $1(7.1 \%)$ \\
\hline No substitution & $3(75 \%)$ & $4(30.7 \%)$ & $0(0 \%)$ \\
\hline Asp83Asn & $0(0 \%)$ & $1(7.7 \%)$ & $8(57.1 \%)$ \\
\hline Ser79Ala & $0(0 \%)$ & $1(7.7 \%)$ & $2(14.4 \%)$ \\
\hline Ser79Phe & $1(25 \%)$ & $5(38.5 \%)$ & $1(7.1 \%)$ \\
\hline Ser79Tyr & $0(0 \%)$ & $2(15.4 \%)$ & $0(0 \%)$ \\
\hline Ser81Phe & $0(0 \%)$ & &
\end{tabular}

As shown in table 6, at Gatfloxacin MIC 2, 4, 8 and 16, 50\%, 66.7\%, 66.7\% and $100 \%$ had no substitution in Gyr B, 50\% and 25\% at MIC 2 and 8 had Ala583Ser substitution. 33.3\% and 8.3\% at MIC 4 and 8 had Arg545Asn substitution.

Regarding Par C, at Gatfloxacin MIC 2, 4, 8 and 16, 75\%, 49.9\%, 25\% and $11.1 \%$ had no substitution in Par C, 16.7\% at MIC 8 had Asp83Asn substitution. Also only $16.7 \%$ at MIC 4 had Ser79Ala substitution. 25\%, 16.7\%, 50\% and 55.6\% at MIC 2, 4, 8 and 16 had Ser79Phe substitution. At MIC 8 and 16, 8.3\% and 33.3\% had Ser79Tyr substitution. Only $16.7 \%$ at MIC 4 had Ser81Phe substitution.

Table (6) MICs and substitutions observed in GyrB and ParC in Gatfloxacinresistant and intermediate $S$. pneumoniae isolates

\begin{tabular}{|c|c|c|c|c|}
\hline Variable (MIC) & 2 & 4 & 8 & 16 \\
\hline \multicolumn{5}{|l|}{ GyrB } \\
\hline No substitution & $2(50 \%)$ & $4(66.7 \%)$ & $8(66.7 \%)$ & $9(100 \%)$ \\
\hline Ala583Ser & $2(50 \%)$ & O (0\%) & $3(25 \%)$ & 0 (0\%) \\
\hline Arg545Asn & ( $(0 \%)$ & $2(33.3 \%)$ & $1(8.3 \%)$ & ( $(0 \%)$ \\
\hline \multicolumn{5}{|l|}{ Par C } \\
\hline No substitution & $3(75 \%)$ & $3(49.9 \%)$ & $3(25 \%)$ & $1(11.1 \%)$ \\
\hline Asp83Asn & $0(0 \%)$ & O $(0 \%)$ & $2(16.7 \%)$ & O $(0 \%)$ \\
\hline Ser79Ala & O (0\%) & $1(16.7 \%)$ & O (0\%) & O (0\%) \\
\hline Ser79Phe & $1(25 \%)$ & $1(16.7 \%)$ & $6(50 \%)$ & $5(55.6 \%)$ \\
\hline Ser79Tyr & $0(0 \%)$ & O(0\%) & $1(8.3 \%)$ & $3(33.3 \%)$ \\
\hline Ser81Phe & $0(0 \%)$ & $1(16.7 \%)$ & O $(0 \%)$ & O (0\%) \\
\hline
\end{tabular}

As shown in table 7, at Moxifloxacin MIC 2, 4, 8 and 16, 100\%, 44.4\%, $10 \%$ and $12.5 \%$ had no substitution in Gyr B, 25\%, 22.2\% and 20\% at MIC 2, 4 and 8 had Ala583Ser substitution. 25\%, 11.1\% and 12.5\% at MIC 2, 4 and 16 had Arg545Asn substitution.

Regarding Par C, at Moxifloxacin MIC 2, 4, 8 and 16, 75\%, 49.9\%, 25\% and 11.1\% had no substitution in Par C, $11.2 \%$ and $10 \%$ at MIC 4 and 8 had Asp83Asn substitution. Also only $10 \%$ at MIC 8 had Ser79Ala substitution. 44.4\%, 50\% and $50 \%$ at MIC 4, 8 and 16 had Ser79Phe substitution. At MIC 8 and 16, 20\% and 25\% had Ser79Tyr substitution. 
SOHAG MEDICAL JOURNAL

Vol. 22 No.1 Jan 2018
Molecular characterization of mutation in the parC

Mohammed H. Mahmoud ${ }^{4}$

Table (7) MICs and substitutions observed in GyrB and ParC in Moxifloxacin-resistant and intermediate $S$. pneumoniae isolates

\begin{tabular}{|c|c|c|c|c|}
\hline Variable (MIC) & 2 & 4 & 8 & 16 \\
\hline GyrB & & & & \\
\hline No substitution & $2(50 \%)$ & $6(66.7 \%)$ & $8(80 \%)$ & $7(87.5 \%)$ \\
\hline Ala583Ser & $1(25 \%)$ & $2(22.2 \%)$ & $2(20 \%)$ & $0(0 \%)$ \\
\hline Arg545Asn & $1(25 \%)$ & $1(11.1 \%)$ & $0(0 \%)$ & $1(12.5 \%)$ \\
\hline Par C & & & & $1(10 \%)$ \\
\hline No substitution & $4(100 \%)$ & $4(44.4 \%)$ & $1(12.5 \%)$ \\
\hline Asp83Asn & $0(0 \%)$ & $1(11.2 \%)$ & $1(10 \%)$ & $0(0 \%)$ \\
\hline Ser79Ala & $0(0 \%)$ & $0(0 \%)$ & $5(50 \%)$ & $4(50 \%)$ \\
\hline Ser79Phe & $0(0 \%)$ & $4(44.4 \%)$ & $2(20 \%)$ & $2(25 \%)$ \\
\hline Ser79Tyr & $0(0 \%)$ & $0(0 \%)$ & & \\
\hline
\end{tabular}

\section{Discussion}

Ninty one $(91 \%)$ of isolates in our study were resistant to ampicillin, $5.1 \%$ were intermediate and $3.8 \%$ were susceptible. Regarding cefaclor $83.3 \%$ were resistant, $7.7 \%$ were intermediate and $9 \%$ were susceptible. Erythromycin was resistant in $82.1 \%$ of isolates, intermediate in $10.3 \%$, and susceptible in $7.7 \%$. Regarding imipenem $10.3 \%$ of isolates were resistant. Tetracycline was resistant in $71.8 \%$ of isolates. Clarithromycin was resistant in only $6.4 \%$, also $10.3 \%$ of our isolates were resistant to ceftriaxone.Trimethoprim/sulfametho xazole was resistant in $9 \%$ of our isolates.

The MICs of Ciprofloxacin, Levofloxacin, Gatifloxacin and Moxifloxacin were measured in this study and we found that, $44.9 \%$ of S. pneumonia isolates were resistant to ciprofloxacin, $11.5 \%$ were intermediate and $43.6 \%$ were sensitive. Regarding levofloxacin $42.3 \%$ of isolates were resistant, 9\% were intermediate, and $48.7 \%$ were sensitive. Over forty six $(46.1 \%)$ of our isolates were resistant to Gatfloxacin, $10.3 \%$ were intermediate, and $43.6 \%$ were sensitive. Regarding Moxifloxacin $46.2 \%$ of our isolates were resistant, $7.6 \%$ were intermediate, and $46.2 \%$ were sensitive. Also in study of Karger et al. (11) the resistance percentages of all strains to tested antibiotics were as follows: ciprofloxacin $73.33 \%$, ofloxacin $53.33 \%$, norfloxacin $48.89 \%$, and levofloxacin $42.22 \%$. The highest resistance was observed in patients in the age group of $31-40$ years ${ }^{(11)}$.

Regarding genetic substitution, we found that at ciprofloxacin MIC 2, 4,8 and $16,75 \%, 66.7 \%, 77.8 \%$ and $80 \%$ had no substitution in Gyr B, $22.2 \%, 11.1 \%$ and $20 \%$ at MIC 4, 8 and 16 had Ala583Ser substitution. $25 \%, 11.1 \%$ and $11.1 \%$ at MIC 2, 4 and 8 had Arg545Asn substitution.

In Par C, at ciprofloxacin MIC 2, 4, 8 and $16,75 \%, 55.6 \%, 33.3 \%$ had no substitution in Par C, $11.1 \%$ and $10 \%$ at MIC 8 and 16 had Asp83Asn substitution. Only $10 \%$ at MIC 16 had Ser79Ala substitution. 25\%, 33.3\%, $33.3 \%$ and $70 \%$ at MIC 2, 4, 8 and 16 had Ser79Phe substitution. At MIC 4, 8 and $16,11.1,22.2 \%$ and $10 \%$ had Ser79Tyr substitution.

Similar to our results, in studies of Bast et al. ${ }^{(12)}$, Broskey et al. ${ }^{(13)}$, Brueggemann et al. (14) the most frequently observed substitutions in ParC were at positions Ser79 (Ala, 
Phe or Tyr) (74\% of ciprofloxacinresistant isolates) and Asp83 (Ala, Asn, Gly or Tyr $) \quad(15 \%$ of ciprofloxacin-

resistantisolates).Overall, the most common genotype observed was Ser79Phe (ParC) $(35 \%$ of ciprofloxacinresistant isolates). The second most common genotype was isolates with a single ParC substitution (Ser79Phe) (13\% of ciprofloxacinresistant isolates). The high prevalence of these substitutions and their association with fluoroquinolone resistance are consistent with observations published by other investigators (Beekmann et al. (15), Jones et al. $\left.{ }^{(16)}\right)$. Also in study of Korzheva et al. (17) substitutions at Ser79 in ParC are believed to be the most commonly observed substitutions as these positions interact with the fluoroquinolone in the ternary complex.

Regarding levofloxacin, we found that at levofloxacin MIC 4, 8 and 16, 75\%, $61.5 \%$ and $85.7 \%$ had no substitution in Gyr B, 25\%, 23.1\% and $7.1 \%$ at MIC 4, 8 and 16 had Ala583Ser substitution. $15.4 \%$ and $7.1 \%$ at MIC 4 and 8 had Arg545Asn substitution. In Par C, at levofloxacin MIC 4, 8 and $16,75 \%, 30.7 \%, 14.3 \%$ had no substitution in Par C, $7.7 \%$ and $7.1 \%$ at MIC 8 and 16 had Asp83Asn substitution. Only $7.7 \%$ at MIC 8 had Ser79Ala substitution. 25\%, $38.5 \%$ and $57.1 \%$ at MIC 4, 8 and 16 had Ser79Phe substitution. At MIC 8 and 16, 15.4 and $14.4 \%$ had Ser79Tyr substitution. Only $7.1 \%$ at MIC 16 had Ser81Phe substitution. On the other hand, previous studies reported that between $59 \%$ and $71 \%$ of isolates with levofloxacin MICs of $2 \mathrm{pg} / \mathrm{mL}$ had QRDR substitutions in ParC (18, 19).

Regarding gatfloxacin, we found that in Gyr B, at gatfloxacin MIC 2, 4, 8 and $16,50 \%, 66.7 \%, 66.7 \%$ and $100 \%$ had no substitution, $50 \%$ and $25 \%$ at MIC 2 and 8 had Ala583Ser substitution. $33.3 \%$ and $8.3 \%$ at MIC 4 and 8 had Arg545Asn substitution. In Par C, at gatfloxacin MIC 2, 4, 8 and $16,75 \%, 49.9 \%, 25 \%$ and $11.1 \%$ had no substitution in Par C, $16.7 \%$ at MIC 8 had Asp83Asn substitution. Also only $16.7 \%$ at MIC 4 had Ser79Ala substitution. 25\%, $16.7 \%$, $50 \%$ and $55.6 \%$ at MIC 2, 4, 8 and 16 had Ser79Phe substitution. At MIC 8 and $16,8.3 \%$ and $33.3 \%$ had Ser79Tyr substitution. Only $16.7 \%$ at MIC 4 had Ser81Phe substitution.

Regarding moxifloxacin, we found that in Gyr B, at moxifloxacin MIC 2, 4,8 and $16,100 \%, 44.4 \%, 10 \%$ and $12.5 \%$ had no substitution, $25 \%$, $22.2 \%$ and $20 \%$ at MIC 2, 4 and 8 had Ala583Ser substitution. 25\%, 11.1\% and $12.5 \%$ at MIC 2, 4 and 16 had Arg545Asn substitution. In Par C, at moxifloxacin MIC 2, 4, 8 and 16, $75 \%, 49.9 \%, 25 \%$ and $11.1 \%$ had no substitution in Par C, $11.2 \%$ and $10 \%$ at MIC 4 and 8 had Asp83Asn substitution. Also only $10 \%$ at MIC 8 had Ser79Ala substitution. 44.4\%, $50 \%$ and $50 \%$ at MIC 4, 8 and 16 had Ser79Phe substitution. At MIC 8 and $16,20 \%$ and $25 \%$ had Ser79Tyr substitution. Only $12.5 \%$ at MIC 16 had Ser81Phe substitution.

In study of Karger et al. (11) investigated the prevalence of mutations in the parC genes and their role in the development of quinolone resistance. Similar to the findings of other investigations, their results showed the highest prevalence of mutations regarded to the parC gene with $75.56 \%$. The prevalence of this gene was reported to be $67.3 \%$ in 2001 in the United States ${ }^{(14)}, 21.9 \%$ in $2005^{(20)}$ and $70 \%$ in 2009 in Italy ${ }^{(21)}$. Sierra et al. ${ }^{(22)}$ recently correlated mutagenic potency of the 
fluoroquinolones to likelihood of mutant selection. They found levofloxacin and moxifloxacin to be less mutagenic than ciprofloxacin and gemifloxacin and resistant mutants to be selected most commonly by ciprofloxacin followed by gemifloxacin, moxifloxacin and levofloxacin ${ }^{(22)}$.

In study of Brino et al. (23) most isolates had mutations at conventional sites in parC (codons for S79 or D83). However, 4 of 16 isolates with highlevel resistance to one or more fluoroquinolones did not contain a mutation in the codon for S79 of ParC, the amino acid position most frequently reported to be associated with resistance of pneumococci to this class of agents. In addition, 3 of these 16 isolates had multiple mutations that included sites in gyrB. Fass et al. ${ }^{(24)}$ found that only the MICs of levofloxacin and ofloxacin were increased with the introduction of this mutation into parE. However, when the same mutation occurred in $\mathrm{gyrB}$, MICs were increased for all fluoroquinolones except moxifloxacin and gemifloxacin. Interestingly, GyrB

\section{Conclusion}

The present study provide an opportunity to view the predominant mutations

\section{Recommandations}

We recommend:

1. Close attention to monitor fluoroquinolone susceptibility patterns and the association of multidrug resistance with fluoroquinolone resistance in isolates of S.pneumoniae.

2. The increased prescription of fluoroquinolones as first-line therapy for common infections such as respiratory tract infection will facilitate the emergence of resistance to this class of compounds and promote the emergence of multidrug-resistant strains and, therefore, should be mutants (changes of either E474K or D435E) were important for resistance to gatifloxacin but not to moxifloxacin. The chemical structures of these 8-methoxyquinolones differ only in their C-7 substituents ${ }^{(25,26)}$.

In addition to gyrB mutations, a novel amino acid change of A63T in ParC was detected in one isolate by Yoshida et al. ${ }^{(27)}$. Unlike most parC mutations in pneumococci, this alteration could not be selected on levofloxacin as a first-round transformant. However, when the parC gene fragment encoding the A63T change was introduced after the 4571 gyrB mutations, MICs of five fluoroquinolones increased two- to fourfold, indicating that mutations affecting this amino acid position make significant contributions to resistance. No alterations have been reported for this amino acid position in S. pneumonia ${ }^{(27)}$. Fukushima et al. (28) also reported the use of melting curve analysis for the detection of QRDR mutations in S. pneumoniae. They used two pairs of probes for the detection of the Ser79 and Asp83 mutations in $\mathrm{ParC}^{(28)}$.

conferring reduced susceptibility to FQs in clinical pneumococcal isolates.

discouraged as it will undermine the efficacy of fluoroquinolones to treat more-serious infections.

3. Continued surveillance of respiratory tract isolates and other pathogens is important, and appropriate clinical use of fluoroquinolones is imperative as they become more widely prescribed.

4. Further studies in larger numbers of patients are necessary to establish the role of gene substitution in (QRDRs) in S. pneumonia isolates and resistance to Fluoroquinlones. 
5. Future prevalence studies will be able to track changes in the predominant mutations conferring resistance to FQs

6. In order to accurately analyze the increasing trend of fluoroquinolone resistance-associated substitutions in fluoroquinolone-susceptible S. pneumoniae isolates, the study of susceptible isolates will need to be

\section{References}

1. Mandell LA, Wunderink RG, Anzueto A, Bartlett JG, Campbell GD, Dean NC, et al. Infectious Diseases Society of America/American Thoracic Society consensus guidelines on the management of community-acquired pneumonia in adults. Clinical infectious diseases : an official publication of the Infectious Diseases Society of America. 2007;44 Suppl 2:S27-72.

2. Patel SN, McGeer A, Melano R, Tyrrell GJ, Green K, Pillai DR, et al. Susceptibility of Streptococcus pneumoniae to fluoroquinolones in Canada. Antimicrobial agents and chemotherapy. 2011;55(8):3703-8.

3. Morrissey I, Farrell DJ, Bakker S, Buckridge S, Felmingham D. Molecular characterization and antimicrobial susceptibility of fluoroquinolone-resistant or susceptible Streptococcus pneumoniae from Hong Kong. Antimicrobial agents and chemotherapy. 2003;47(4):1433-5.

4. de la Campa AG, Balsalobre L, Ardanuy C, Fenoll A, Perez-Trallero $\mathrm{E}$, Linares $\mathrm{J}$, et al. Fluoroquinolone resistance in penicillin-resistant Streptococcus pneumoniae clones, Spain. Emerging infectious diseases. 2004;10(10):1751-9.

5. Fisher LM, Gould KA, Pan XS, Patel $\mathrm{S}$, Heaton VJ. Analysis of dual active fluoroquinolones in Streptococcus repeated in a few years in different and variable hospitals and clinics in our country.

7. The maintenance of such surveillance is valuable in the preparation of future therapy guidelines and could lead to new therapeutic strategies for FQ-resistant S.Pneumoniae. pneumoniae. The Journal of antimicrobial chemotherapy. 2003;52(2):312-3; author reply 3-4.

6. Andersson MI, MacGowan AP. Development of the quinolones. The Journal of antimicrobial chemotherapy. 2003;51 Suppl 1:1-11. 7. Collins MD, Hutson RA, Hoyles L, Falsen E, Nikolaitchouk N, Foster G. Streptococcus ovis sp. nov., isolated from sheep. International journal of systematic and evolutionary microbiology. 2001;51(Pt 3):1147-50.

8. Zhanel GG, Palatnick L, Nichol KA, Bellyou T, Low DE, Hoban DJ. Antimicrobial resistance in respiratory tract Streptococcus pneumoniae isolates: results of the Canadian Respiratory Organism Susceptibility Study, 1997 to 2002. Antimicrobial agents and chemotherapy. 2003;47(6):1867-74.

9. Tettelin H, Nelson KE, Paulsen IT, Eisen JA, Read TD, Peterson S, et al. Complete genome sequence of a virulent isolate of Streptococcus pneumoniae. Science. 2001;293(5529):498-506.

10. Hoskins J, Alborn WE, Jr., Arnold J, Blaszczak LC, Burgett S, DeHoff BS, et al. Genome of the bacterium Streptococcus pneumoniae strain R6. Journal of bacteriology. 2001;183(19):5709-17.

11. Kargar M, Moein Jahromi F, Doosti A, Handali S. Molecular Investigation 
of Quinolone Resistance of Quinolone Resistance-Determining Region in Streptococcus pneumoniae Strains Isolated from Iran Using Polymerase Chain Reaction-Restriction Fragment Length Polymorphism Method. Osong public health and research perspectives. 2014;5(5):245-50.

12. Bast DJ, Low DE, Duncan CL, Kilburn L, Mandell LA, Davidson RJ, et al. Fluoroquinolone resistance in clinical isolates of Streptococcus pneumoniae: contributions of type II topoisomerase mutations and efflux to levels of resistance. Antimicrobial agents and chemotherapy. 2000;44(11):3049-54.

13. Broskey J, Coleman K, Gwynn MN, McCloskey L, Traini C, Voelker L, et al. Efflux and target mutations as quinolone resistance mechanisms in clinical isolates of Streptococcus pneumoniae. The Journal of antimicrobial chemotherapy. 2000;45 Suppl 1:95-9.

14. Brueggemann AB, Coffman SL, Rhomberg P, Huynh $\mathrm{H}$, Almer L, Nilius A, et al. Fluoroquinolone resistance in Streptococcus pneumoniae in United States since 1994-1995. Antimicrobial agents and chemotherapy. 2002;46(3):680-8.

15. Beekmann SE, Heilmann KP, Richter SS, Garcia-de-Lomas J, Doern GV, Group GS. Antimicrobial resistance in Streptococcus pneumoniae, Haemophilus influenzae, Moraxella catarrhalis and group A betahaemolytic streptococci in 2002-2003. Results of the multinational GRASP Surveillance Program. International journal of antimicrobial agents. 2005;25(2):148-56.

16. Jones ME, Sahm DF, Martin N, Scheuring S, Heisig P, Thornsberry C, et al. Prevalence of gyrA, gyrB, parC, and parE mutations in clinical isolates of Streptococcus pneumoniae with decreased susceptibilities to different fluoroquinolones and originating from Worldwide Surveillance Studies during the 1997-1998 respiratory season. Antimicrobial agents and chemotherapy. 2000;44(2):462-6.

17. Korzheva N, Davies TA, Goldschmidt R. Novel Ser79Leu and Ser81Ile substitutions in the quinolone resistance-determining regions of ParC topoisomerase IV and GyrA DNA gyrase subunits from recent fluoroquinolone-resistant

Streptococcus pneumoniae clinical isolates. Antimicrobial agents and chemotherapy. 2005;49(6):2479-86.

18. Davies TA, Evangelista A, Pfleger S, Bush K, Sahm DF, Goldschmidt R. Prevalence of single mutations in topoisomerase type II genes among levofloxacin-susceptible clinical strains of Streptococcus pneumoniae isolated in the United States in 1992 to 1996 and 1999 to 2000. Antimicrobial agents and chemotherapy. 2002;46(1):119-24.

19. Lim S, Bast D, McGeer A, de Azavedo J, Low DE. Antimicrobial susceptibility breakpoints and firststep parC mutations in Streptococcus pneumoniae: redefining fluoroquinolone resistance. Emerging infectious diseases. 2003;9(7):833-7.

20. Doern GV, Richter SS, Miller A, Miller N, Rice C, Heilmann K, et al. Antimicrobial resistance among Streptococcus pneumoniae in the United States: have we begun to turn the corner on resistance to certain antimicrobial classes? Clinical infectious diseases : an official publication of the Infectious Diseases Society of America. 2005;41(2):13948.

21. De Vecchi E, Nicola L, Ossola F, Drago L. In vitro selection of resistance in Streptococcus pneumoniae at in vivo fluoroquinolone concentrations. The 
SOHAG MEDICAL JOURNAL

Vol. 22 No.1 Jan 2018
Journal of antimicrobial chemotherapy. 2009;63(4):721-7.

22. Sierra JM, Cabeza JG, Ruiz Chaler M, Montero T, Hernandez J, Mensa J, et al. The selection of resistance to and the mutagenicity of different fluoroquinolones in Staphylococcus aureus and Streptococcus pneumoniae. Clinical microbiology and infection : the official publication of the European Society of Clinical Microbiology and Infectious Diseases. 2005;11(9):750-8.

23. Brino L, Urzhumtsev A, Mousli M, Bronner C, Mitschler A, Oudet P, et al. Dimerization of Escherichia coli DNA-gyrase B provides a structural mechanism for activating the ATPase catalytic center. The Journal of biological chemistry. 2000;275(13):9468-75.

24. Fass D, Bogden CE, Berger JM. Quaternary changes in topoisomerase II may direct orthogonal movement of two DNA strands. Nature structural biology. 1999;6(4):322-6.

25. Hosaka M, Kinoshita S, Toyama A, Otsuki M, Nishino T. Antibacterial properties of AM-1155, a new 8methoxy quinolone. The Journal of antimicrobial chemotherapy. 1995;36(2):293-301.

26. Stass H, Dalhoff A, Kubitza D, Schuhly U. Pharmacokinetics, safety, and tolerability of ascending single doses of moxifloxacin, a new 8methoxy quinolone, administered to healthy subjects. Antimicrobial agents and chemotherapy. 1998;42(8):20605.

27. Yoshida H, Bogaki M, Nakamura M, Nakamura S. Quinolone resistancedetermining region in the DNA gyrase gyrA gene of Escherichia coli. Antimicrobial agents and chemotherapy. 1990;34(6):1271-2.

28. Fukushima KY, Hirakata Y, Sugahara K, Yanagihara K, Kondo A, Kohno S, et al. Rapid screening of topoisomerase gene mutations by a novel melting curve analysis method for early warning of fluoroquinoloneresistant Streptococcus pneumoniae emergence. Journal of clinical microbiology. 2006;44(12):4553-8. 